\title{
LA MOVILIDAD DE LOS RANKINGS DE HOTELES EN EUROPA EL CASO DE TRIPADVISOR
}

\author{
José Luis Ximénez-de-Sandoval \\ Universidad de Málaga (España) \\ Facultad de Turismo \\ joseluis.xs@uma.es \\ Antonio Guevara Plaza \\ Universidad de Málaga (España) \\ Facultad de Turismo \\ guevara@uma.es
}

Antonio Fernández-Morales

Universidad de Málaga (España)

Facultad de CC. Económicas y Empresariales afdez@uma.es

(Fecha envío: 20/11/16 - Fecha aceptación: 20/05/17)

\section{Resumen}

La influencia que el ranking de hoteles de Tripadvisor ejerce en los turistas es reconocida tanto en el mundo académico como empresarial.

Pese a la gran cantidad de autores que han analizado esta aplicación desde múltiples ángulos, nuestro trabajo se centra en un aspecto que todavía no ha sido estudiado: La evolución temporal del ranking. En sep/2015 iniciamos la extracción de las clasificaciones de los hoteles de las capitales europeas. En mar/2016 volvimos a extraer los rankings, comparando la evolución en el tiempo de más de 8.000 hoteles para responder entre otras a las siguientes cuestiones: ¿Cómo varía el ranking a lo largo del tiempo? ¿Los mejores hoteles en 2015 siguen siendo los mismos en 2016? ¿Hay grandes saltos en la clasificación? Los sorprendentes resultados obtenidos nos indican que Tripadvisor, como consecuencia de su enorme éxito y de su propio modo de establecer las valoraciones de los hoteles, está llegando al punto de saturación, al colapso.

\section{Palabras clave}

Tripadvisor, ranking, hoteles, comentarios, opiniones, reputación online, valoración de hoteles, clasificación de hoteles

\section{Abstract}

The influence that the Tripadvisor Hotels Ranking has on tourists when deciding where to stay is recognized by both the academic and business world.

Despite the large number of authors who have analyzed this application from multiple angles, our work focuses on an aspect that is still little studied: Ranking Mobility.

In Sep/2015 we started this work by collecting hotels rankings in the capitals of the 28 countries of the EU. In Mar/2016 we extracted again the rankings, comparing the evolution over time of the ranking positions of more than 8,000 European hotels to answer the following questions:

How do the ranking positions evolve over time? Did the hotels best positioned in 2015 keep their positions in 2016 ? Is it the same at the bottom of the list? Have any of the hotels made a big jump up or down within the list?

Our surprising results indicate that Tripadvisor's hotel ranking, due to both its enormous success and its specific way to sort out hotels, is reaching saturation point.

\section{Keywords}

Tripadvisor, ranking, mobility, online reviews, user generated content, hotels ranking systems, data mining, online reputation 


\section{INTRODUCCION}

Las listas son útiles. Nos ayudan a tomar decisiones. Por eso los seres humanos nos hemos sentido atraídos por ellas desde tiempos remotos. Así nos lo indica Umberto Eco (2009) cuando nos recuerda que ya en la llíada de Homero encontramos ejemplos de listas. También considera el pensador italiano que la gran madre de todas las listas es la World Wide Web, infinita por definición al estar en continuo desarrollo.

En el año dos mil, surge en esta red que llamamos Internet una empresa que ha revolucionado la industria del turismo, precisamente por sus listas: Tripadvisor, que clasifica hoteles y restaurantes a partir de los comentarios y valoraciones de los clientes.

Su capacidad para influir en los turistas a la hora de elegir el hotel donde alojarse es innegable. También es ampliamente reconocida su influencia sobre los empresarios, preocupados porque un comentario negativo pueda dañar su reputación, o una mala posición en el ranking pueda afectar a su cuenta de resultados.

Aunque hay en la literatura científica de los últimos años innumerables trabajos dedicados a analizar desde los más variados puntos de vista esta aplicación, todavía encontramos algunos aspectos escasamente tratados. Uno de ellos será el objeto de nuestro estudio: La movilidad de los rankings, esto es, la evolución a lo largo del tiempo de las posiciones que ocupan los hoteles en la clasificación.

Para entender la importancia de nuestro objeto de estudio, basta con pensar por un instante las implicaciones que tendría para clientes y/o empresarios un ranking por ejemplo en el que apenas se producen cambios en la clasificación a lo largo del tiempo, y en consecuencia, la mayoría de los hoteles de las zonas alta, media y baja conservan las mismas posiciones mes tras mes. Tendríamos entonces que preguntarnos si los clientes y usuarios estarían tan interesados en escribir comentarios si supieran que sus valoraciones (positivas o negativas) apenas iban a influir en el puesto que ocupa en la clasificación el hotel o restaurante en cuestión. También sería interesante conocer si los empresarios seguirían tan preocupados por mejorar su posición en el ranking si supieran que son mínimas, casi nulas, sus posibilidades de alcanzar a medio plazo los mejores puestos, o si utilizarían la posición en la clasificación como incentivo o medida del desempeño de sus empleados.

Nuestro trabajo está justificado en primer lugar por la enorme influencia (reconocida por académicos y empresarios) que Tripadvisor ejerce en la industria turística, lo que nos obliga como investigadores, en un ejercicio de responsabilidad, a tratar de aportar luz sobre el funcionamiento de estos rankings. En segundo lugar, por las importantes implicaciones que una mayor o menor movilidad puede tener para los distintos actores implicados (clientes, empresarios, trabajadores, destinos). Y en tercer lugar por la escasez de trabajos sobre esta materia.

Por todo ello, el objetivo fundamental de nuestra investigación es analizar cómo varía la clasificación de hoteles de Tripadvisor, en concreto los rankings de las 28 ciudades capitales de los estados miembros de la UE, para responder entre otras a las siguientes cuestiones:

(1) ¿Los mejores hoteles en 2015 siguen siendo los mismos en 2016?, (2) ¿Y los peores hoteles, también siguen siendo los mismos?, (3) ¿Qué ocurre con los hoteles situados en las zonas centrales del ranking?, ¿tienden a subir hacia mejores posiciones, a bajar hacia la parte inferior o bien se mantienen en la zona central? (4) ¿Hay diferencias significativas entre las distintas ciudades en las variaciones de sus rankings?, (5) ¿Hay muchos hoteles que presenten grandes saltos en la clasificación o por el contrario son pocas las posiciones que un hotel sube o baja en seis meses?

La estructura de nuestro trabajo es la siguiente: En primer lugar, realizamos una revisión bibliográfica sobre Tripadvisor y los rankings de hoteles, posteriormente detallaremos la metodología aplicada, así como los pasos seguidos en la construcción de nuestra base de datos. A continuación, presentamos los resultados obtenidos, repartidos en tres apartados; (1) Análisis de los cambios en la clasificación, (2) análisis de los cambios en las puntuaciones de los hoteles y (3) análisis de los cambios en el número de comentarios recibidos. Por último, planteamos las principales conclusiones y una serie de sugerencias, junto con la bibliografía utilizada.

\section{REVISION DE LA LITERATURA}

Tanto a nivel académico como profesional, está ampliamente aceptada la idea de que las redes sociales juegan un papel cada vez más importante como fuente de información para los viajeros (Xiang \& Gretzel, 2010) en el proceso de búsqueda para la compra de un viaje (Jain et al. 2012) De hecho, y desde una perspectiva histórica estamos viviendo una nueva era en las relaciones entre la tecnología y el turismo (Mistilis \& Buhalis, 2012).

El fenómeno de las opiniones de los usuarios en Internet ha cambiado la manera en que los consumidores compran productos y servicios (Ghose et al., 2012). Hoy día quien esté planificando un viaje, tiene más confianza en los comentarios de otros turistas que en los consejos de una agencia de viajes (Ong, 2012). 
Tripadvisor como fuente de información para los investigadores puede ser considerada desde dos puntos de vista. En primer lugar, como repositorio de comentarios, fotografías y valoraciones individuales de clientes de hoteles y restaurantes. Pero además, Tripadvisor, haciendo uso de aquellas opiniones y valoraciones, es también un creador de rankings.

Pues bien, mientras que la inmensa mayoría de investigadores han estudiado el fenómeno Tripadvisor como almacén de opiniones y valoraciones individuales y por tanto como típico ejemplo de e-WOM (Chen \& Law, 2016; Sotiriadis \& Sotiriadis, 2017), son muy pocos, por contra, los trabajos que se han ocupado de Tripadvisor como clasificación de hoteles.

Es aquí donde en nuestra opinión hay un gran campo por explorar, no sólo en el apartado en el que nuestra investigación se enmarca (movilidad de los rankings) sino en otros ámbitos de enorme importancia, ya sean relacionados con el marketing (Tripadvisor como ejemplo de Publicity para hoteles y restaurantes), en el ámbito de la auditoría y la responsabilidad social (quién audita, controla o verifica el funcionamiento de estos rankings), en aspectos relacionados con la tecnología y los sistemas de información (cómo funciona el algoritmo que crea los rankings, o en qué medida son similares/comparables los rankings de hoteles de distintas plataformas como Tripadvisor o Booking).

Uno de los primeros trabajos que analiza la web de Tripadvisor no como depósito de opiniones sino como ranking, se debe a Smyth et al. (2010). Estos autores analizaron más de 30.000 comentarios sobre hoteles de Irlanda, y los comparan con 50.000 comentarios de hoteles de Las Vegas durante dos años. Mientras la puntuación media de los hoteles de Las Vegas se había mantenido constante en torno a 3,8 puntos, los hoteles irlandeses pasaron de una puntuación media de 3,6 a 3,8. Para los autores esto es debido a lo que ellos llaman "EI Efecto TripAdvisor". Mientras los hoteles en Las Vegas hace ya tiempo que alcanzaron unos aceptables niveles de calidad, los hoteleros irlandeses, siendo conscientes de que unos comentarios negativos en TripAdvisor podían dañar la imagen de sus establecimientos, han trabajado para mejorar sus niveles de servicio y así evitar futuros comentarios negativos.

En este sentido, hay evidencias de que las puntuaciones de los clientes tienen un fuerte impacto en la intención de reservar un hotel. Cuando un establecimiento tiene comentarios negativos Verma et al. (2012) señalan que hay una probabilidad de $40 \%$ de reservar en ese hotel. Cuando el comentario es positivo la probabilidad sube al 70-80\%. Melián et al. (2010), recomiendan a los directores de hotel que fomenten la participación de sus clientes ya que, según estos autores, cuantas menos valoraciones recibe un hotel más negativas son las mismas.

Un apartado que está generando un número importante de estudios es la reputación online. La importancia de los comentarios para el valor de la marca ha sido estudiada por Callarisa-Fiol et al. (2012). Analizando sólo las puntuaciones cuantitativas de turistas que habían opinado en TripAdvisor sobre sus estancias en hoteles de París y Hong Kong, los autores observan que los de la primera ciudad son más recomendados que los de la segunda, interpretando esto como un signo de mayor satisfacción.

Stringam et al. (2010) encuentran evidencias de que la mayoría de los viajeros que escriben comentarios sobre un hotel estarían dispuestos a recomendarlo (74,51\%), y de que la mayoría valora positivamente su experiencia. Concretamente el $72,85 \%$ dio a los hoteles una puntuación entre 4 y 5 puntos sobre 5 , mientras que sólo un $13,43 \%$ de los viajeros asignan un 1 o un 2 en cuanto a su nivel de satisfacción general.

Al ser la puntuación global de un hotel un promedio de todas las puntuaciones individuales recibidas por ese establecimiento, Jurca et al. (2010) se preguntan cómo cambiarían dichas puntuaciones globales si en vez de la media aritmética se usara la mediana o la moda. Utilizando la mediana, la posición en el ranking difiere por término medio 7,7 posiciones, y utilizando la moda 16,9 posiciones respecto a las calculadas con media aritmética. Para estos autores la mediana y la moda proporcionan resultados más robustos frente a comentarios que intencionadamente puedan distorsionar la puntuación global de un hotel.

El problema del exceso de información a disposición del usuario es reconocido por O'Mahony et al (2010) quienes entienden que hay una gran producción de opiniones por parte de los usuarios pero que un gran número de ellas no son de interés. Esta gran cantidad de comentarios publicados por los turistas ha generado también gran preocupación en el sector ante la posibilidad de fraude. Ong (2012) se preguntó si la cantidad de comentarios afecta a la fiabilidad percibida de los mismos, concluyendo que efectivamente un alto número de opiniones proporciona credibilidad. Quizás los usuarios son conscientes de la posibilidad de que algunos comentarios sean falsos, pero cuando el número de comentarios es elevado (cientos) entonces el impacto de la manipulación de un comentario falso disminuye drásticamente.

Wu et al. (20110) presentan un conjunto de criterios para detectar comentarios sospechosos de ser falsos. Ya que la decisión de compra está cada vez más influida por los comentarios online de los consumidores (Ott et al., 2012) es lógico que exista cierta preocupación ante la posibilidad de encontrar comentarios falsos, esto es, falsas opiniones deliberadamente escritas para parecer auténticas con la intención de engañar al lector (Ott, et al, 2011). Ott et al. (2012) utilizan un modelo para explorar el predominio de la falsedad de los comentarios positivos (no analiza la posibilidad de comentarios negativos falsos) en seis populares comunidades de opiniones online, 
concluyendo que los sitios con una baja dificultad para escribir comentarios tienen un mayor nivel de falsos comentarios. Sus resultados señalan que TripAdvisor estaría en torno al $4 \%$ de comentarios fraudulentos.

Fruto de esta preocupación por el fraude, en 2014 la Comisión Europea (EC 2014) publicó un informe específico sobre los comentarios online de los clientes en el sector hotelero donde entre otras recomendaciones indicaba que las webs de opiniones deben tomar medidas para verificar que sus usuarios se alojaron realmente en el establecimiento sobre el que están opinando.

En este sentido, en diciembre de 2014 la ICA (Italian Competition Authority) sancionó con una multa de 500.000 Euros a Tripadvisor por infringir varios artículos del código de comercio italiano (Biffaro 2015). El ICA encontró desleal la falta de medidas por parte de Tripadvisor para atajar las opiniones engañosas. Pero, como indica, (Hensens 2010) la mayor medida que tiene Tripadvisor para luchar contra los comentarios fraudulentos es el elevado número de opiniones por hotel. Su investigación sobre el fraude en los comentarios refleja que a partir de cien opiniones un hotel es prácticamente inmune a los comentarios fraudulentos.

En relación al funcionamiento de los rankings de hoteles y al margen de las opiniones falsas o fraudulentas también encontramos otras preocupaciones e inquietudes. Así, Mellinas et al (2015) señalan claramente la falta de honestidad para con los consumidores que presenta el ranking de hoteles de Booking.com al encontrar evidencias de que se manipulan las puntuaciones. También analizan los rankings Mateos et al (2015) quienes en este caso llaman la atención sobre el excesivo poder de Booking y la preocupación del sector sobre esta posición dominante.

Por último, Sott \& Orlikowsky (2012), señalan que para ciertas empresas del sector turístico una mejor o peor calificación en el ranking puede suponer la diferencia entre las ganancias o las pérdidas. Por tanto, es necesario tomar en serio las relaciones de responsabilidad que se producen en TripAdvisor, ya que el resultado es en la práctica una nueva especie de autoridad que dirige nuestras vidas. Finalmente subrayan estos autores la paradoja de que TripAdvisor está configurado de tal manera que produce una forma de trasparencia para los comentarios de los turistas mientras que oscurece el mecanismo que ordena esos comentarios.

\section{METODOLOGÍA Y BASE DE DATOS}

Nuestro trabajo se encuadra dentro de un amplio proyecto de investigación que pretende monitorizar periódicamente el funcionamiento y la evolución de los rankings de hoteles y restaurantes en las principales ciudades de los cinco continentes en Tripadvisor y Booking, las dos principales plataformas de valoración de hoteles a partir de comentarios de usuarios sobre establecimientos turísticos.

El proceso de extracción de datos lo iniciamos en junio de 2015 cuando recopilamos las clasificaciones en Tripadvisor de los hoteles de las ciudades capitales de los 28 estados miembros de la UE. Durante el primer trimestre de 2016, seis meses desde la primera toma de datos, una vez transcurrido tiempo suficiente para poder obtener una primera aproximación sobre la movilidad de estos rankings, volvimos a extraer las clasificaciones en dichas ciudades. Posteriormente se inició el proceso de comparación de datos.

Para realizar nuestra investigación, hemos utilizado una aplicación para la extracción automatizada de datos de la web, una base de datos donde almacenar toda la información recopilada y una hoja de cálculo. Las técnicas estadísticas utilizadas han sido principalmente el análisis de matrices de transición, tablas de contingencia y análisis gráfico-exploratorio.

Al extraer las clasificaciones en las 28 ciudades en junio de 2015, obtuvimos información sobre un total de 9.150 hoteles. En el segundo periodo de extracción, (febrero de 2016) recopilamos información de 9.215 hoteles.

Tras eliminar los no coincidentes, la muestra final consta de 8.145 hoteles que serán objeto de nuestro estudio comparando su posición en 2015 y 2016. Esto supone el $89 \%$ de los hoteles que teníamos en 2015 y el $88,4 \%$ de los hoteles que tenemos en 2016.

Pese a tener que renunciar a un $11 \%$ de nuestros datos, creemos que el número de hoteles con el que contamos para nuestro análisis (8.145) es suficientemente elevado como para que nuestros resultados sean válidos.

Para cada uno de estos 8.145 hoteles disponemos de la siguiente información: (1) Nombre del hotel, (2) Ciudad, (3) Posición en el Ranking 2015, (4) Posición en el Ranking 2016, (5) Puntuación del hotel en 2015, (6) Puntuación del hotel en 2016, (7) № de comentarios en 2015, (8) № de comentarios en 2016. 
La lista de las 28 ciudades con el número de hoteles analizados se muestra a continuación:

\begin{tabular}{|c|l|c|}
\hline Cod & \multicolumn{1}{|c|}{ Capital } & Hoteles \\
\hline 1 & Paris & 1641 \\
\hline 2 & Roma & 1114 \\
\hline 3 & Londres & 976 \\
\hline 4 & Berlin & 606 \\
\hline 5 & Praga & 593 \\
\hline 6 & Madrid & 395 \\
\hline 7 & Viena & 332 \\
\hline 8 & Amsterdam & 331 \\
\hline 9 & Budapest & 252 \\
\hline 10 & Atenas & 248 \\
\hline 11 & Lisboa & 210 \\
\hline 12 & Bruselas & 185 \\
\hline 13 & Dublin & 157 \\
\hline 14 & Estocolmo & 142 \\
\hline
\end{tabular}

\begin{tabular}{|c|l|c|}
\hline Cod & \multicolumn{1}{|c|}{ Capital } & Hoteles \\
\hline 15 & Bucarest & 134 \\
\hline 16 & Varsovia & 106 \\
\hline 17 & Sofia & 104 \\
\hline 18 & Copenhague & 101 \\
\hline 19 & Riga & 92 \\
\hline 20 & Bratislava & 82 \\
\hline 21 & Tallin & 69 \\
\hline 22 & Vilna & 63 \\
\hline 23 & Zagreb & 53 \\
\hline 24 & Helsinki & 50 \\
\hline 25 & C.de Luxemburgo & 49 \\
\hline 26 & Liubliana & 28 \\
\hline 27 & Nicosia & 24 \\
\hline 28 & La Valeta & 8 \\
\hline
\end{tabular}

Tabla 1: Ciudades y Hoteles en la Muestra

Fuente: Elaboración propia a partir de los datos extraídos de Tripadvisor

\section{VARIACION EN EL RANKING}

El estudio de la variación de las posiciones que los hoteles ocupan en los rankings de estas ciudades a lo largo del tiempo lo hemos dividido en tres apartados:

1- Un primer apartado donde analizamos cuantos hoteles han cambiado de posición en el periodo estudiado. En este sentido, llamamos Volatilidad del ranking al número de hoteles que han cambiado de posición. Un ranking muy volátil será aquel en el que la gran mayoría de hoteles han modificado su posición, entre el momento inicial y el final.

2- Un segundo apartado donde analizamos cuanto han variado los hoteles. Así, llamamos Flexibilidad del ranking al no de posiciones que por término medio los hoteles suben o bajan en la clasificación entre el momento inicial y el final.

3- Por último, realizaremos un análisis de los rankings por zonas, dividiendo la clasificación en zona alta, media y baja para comprobar si el comportamiento es similar en dichas zonas, y estudiar además en qué medida hay trasvase de hoteles de unas zonas a otras. Dada la repercusión que puede tener para un hotel estar en la zona alta del ranking (en términos de reputación, visibilidad o incluso ventas) hablamos en este caso de análisis de la Relevancia de los cambios en el ranking.

\subsection{Volatilidad del ranking: ¿Cuántos hoteles suben o bajan?}

La tabla 2 recoge el número de hoteles que han subido, que no han variado, o que han bajado algún puesto en la clasificación de su ciudad en el periodo analizado.

\begin{tabular}{|c|c|c|c|c|}
\hline Variación en el ranking & Suben & Igual & Bajan & Total \\
\hline Hoteles & 2813 & 278 & 5054 & 8.145 \\
\hline$\%$ & $34,54 \%$ & $3,41 \%$ & $62,05 \%$ & $100 \%$ \\
\hline
\end{tabular}

Tabla 2: Volatilidad del ranking

\footnotetext{
${ }^{1}$ Todos los cuadros, tablas y gráficos que aparecen en este trabajo son de elaboración propia a partir de los datos extraídos de Tripadvisor.
} 
Vemos que tan sólo el 3,4 \% de los hoteles no han variado su posición en el ranking. Además, hay una diferencia muy significativa entre el número de hoteles que han bajado algún puesto en el ranking (62,05\%) y los que han subido en la clasificación (34,53\%). Podemos por tanto concluir diciendo que casi la totalidad de los hoteles $(96,59 \%=34,54 \%+62,05 \%)$ ha variado su posición en el ranking en los últimos seis meses.

\subsection{Flexibilidad del Ranking: ¿Cuánto suben o bajan los hoteles?}

Hemos visto que la mayoría de hoteles varía su posición, pero ¿cuántos puestos suben o bajan? El siguiente cuadro nos resume las principales cifras relacionadas con la flexibilidad de los rankings:

\begin{tabular}{|c|c|c|c|c|}
\hline Variación en el ranking & Hoteles & Distancia máxima & Distancia mínima & Distancia promedio \\
\hline Bajadas & 5054 & 787 & 1 & $-35,83$ \\
\hline Subidas & 2813 & -682 & -1 & 35,96 \\
\hline
\end{tabular}

Tabla 3: Flexibilidad del ranking

Encontramos algún hotel que ha llegado a ascender 787 posiciones en 6 meses y otro que ha caído 682 puestos. No obstante, el promedio de subidas o bajadas es similar (entre 35 y 36 puestos).

Para eliminar el efecto tamaño de la ciudad (no puede tener el mismo valor subir 30 puestos en Roma (1.114 hoteles) que en Helsinki (50 Hoteles), hemos calculado el Ratio R de Flexibilidad del ranking:

$$
R=\frac{\text { PosiciónIncial }- \text { Final }}{N^{\mathbf{o}} \text { Totalde Hoteles }}
$$

donde un valor de $\mathrm{R}$ próximo a 1 indicaría que el hotel ha subido de los últimos puestos a los primeros, mientras que un valor próximo a -1 indicaría que el hotel habría bajado desde las primeras a las últimas posiciones. Por último, los valores cercanos a 0 indicarían que la variación en el ranking ha sido insignificante. El siguiente cuadro nos resume las principales cifras relacionadas con esta ratio:

\begin{tabular}{|c|c|c|c|c|}
\hline Variación en el ranking & Hoteles & Ratio R (Máximo) & Ratio R (Mínimo) & Ratio R (Promedio) \\
\hline Subidas & 2813 & 0,75893 & 0,00057 & 0,04958 \\
\hline Bajadas & 5054 & $-0,64286$ & $-0,00057$ & $-0,04477$ \\
\hline
\end{tabular}

Tabla 4: Ratio $R$ de Flexibilidad del ranking

Aunque algún hotel llega a ascender el $75,8 \%$ del ranking y otro llega a bajar un $64,2 \%$, observamos que, en términos porcentuales las variaciones promedio tanto en las subidas $(4,95 \%)$ como en las bajadas $(-4,47 \%)$ son muy similares y de escasa cuantía.

\subsection{Variaciones en el ranking por zonas}

Ahora debemos preguntarnos si estas variaciones provocan cambios significativos en la clasificación, si tienen en definitiva, Relevancia en términos de pérdidas o ganancias de notoriedad, prestigio y reputación y en última instancia ventas.

Queremos responder a la pregunta que muchos hoteleros se hacen: ¿Puede un hotel en la zona media del ranking llegar a situarse algún día entre los mejores? ¿Puede un hotel situado en la zona alta de la clasificación caer a las zonas medias o bajas? Trataremos además de dar respuesta a las siguientes cuestiones: ¿Cuántos hoteles que estaban entre los mejores en 2015 siguen estando entre los mejores en 2016?, ¿Cuántos hoteles que estaban en los peores puestos en 2015 siguen estando entre los peores en 2016?, ¿De los hoteles que estaban en la zona central del ranking, cuantos han subido a lo más alto o han caído a la parte baja de la clasificación?

\subsubsection{Top-5, Top-10, Top-30}

Analizamos a continuación los cambios en los rankings, pero estudiando los comportamientos en las dos zonas con más importancia en la clasificación: La zona alta (Top) y la zona baja (Peor). Al ser conceptos relativamente imprecisos, hemos delimitado estas zonas de tres maneras distintas, analizando todos los casos.

Dado que en términos comerciales y en publicidad se recurre con frecuencia a resaltar los cinco mejores o también los diez mejores hoteles, analizaremos estas zonas, (Top-5 y Top-10). Además, ya que Tripadvisor 
presenta los resultados en páginas con un máximo de treinta hoteles, analizaremos también el Top-30, de gran relevancia dados los hábitos de los internautas reacios a navegar más allá de las primeras páginas.

La Tabla 5 nos responde a algunas de las cuestiones planteadas más arriba:

\begin{tabular}{|c|c|c|c|c|c|c|}
\hline & Top-5 & Top-10 & Top-30 \\
\hline Promedio & $78,0 \%$ & $84,4 \%$ & $88,7 \%$ \\
\hline Máximo & $100,0 \%$ & $100,0 \%$ & $96,7 \%$ \\
\hline Mínimo & $60,0 \%$ & $70,0 \%$ & $72,4 \%$ \\
\hline
\end{tabular}

Tabla 5: Porcentajes de hoteles que permanecen en las zonas altas y bajas

Como podemos observar, la gran mayoría de hoteles permanecen en la misma zona seis meses después. Esto es debido a que como comprobamos en el análisis de la flexibilidad, los cambios en las posiciones son poco significativos y por tanto sin repercusión en la clasificación a efectos de relevancia. El $78 \%$, el $84,4 \%$ y el $88,7 \%$ de los hoteles que estaban situados en 2015 en el Top-5, Top-10 y Top-30 respectivamente, siguen estando en la misma zona en 2016 .

Al igual que en las zonas altas, la gran mayoría de los hoteles de las capitales europeas situados en las zonas bajas siguen en la misma zona seis meses después. No obstante, es ligeramente inferior el porcentaje de hoteles que permanecen en las zonas bajas en comparación con los de las zonas altas. El $73 \%, 75 \%$ y el $83,1 \%$ de los hoteles que estaban situados respectivamente en los 5, 10 y 30 últimos puestos en 2015, siguen en las peores posiciones en 2016.

Como muestran los valores máximos, en alguna ciudad no se ha producido ni un solo cambio durante el periodo analizado, ya que el $100 \%$ de los hoteles seguía en la misma zona seis meses después.

En cuanto a los valores mínimos, en las zonas altas ninguna ciudad está por debajo del $60 \%$, siendo algo inferior $(40 \%)$ en la zona baja.

\subsubsection{Top-30\%, Centro-40\%, Peor $-30 \%$}

Para eliminar el efecto tamaño de la ciudad, (el puesto 40 no puede tener el mismo valor en Paris con 1.641 hoteles que en Zagreb con 52 Hoteles), y para poder estudiar la evolución de la zona central, vamos a analizar también las variaciones de los hoteles en el ranking no ya por posiciones concretas sino por percentiles.

Utilizamos una matriz de transición para mostrar las variaciones producidas entre la posición que ocupaban los hoteles en el momento inicial (filas) y la posición que ocupan seis meses después (columnas)

\begin{tabular}{|c|c|c|c|}
\cline { 2 - 4 } \multicolumn{1}{c|}{} & $\begin{array}{c}\text { Top-30\% } \\
2016\end{array}$ & $\begin{array}{c}\text { Centro-40\% } \\
2016\end{array}$ & $\begin{array}{c}\text { Peor-30\% } \\
2016\end{array}$ \\
\hline $\begin{array}{c}\text { Top-30\% } \\
2015\end{array}$ & $91,26 \%$ & $8,70 \%$ & $0,04 \%$ \\
\hline $\begin{array}{c}\text { Centro-40\% } \\
2015\end{array}$ & $4,53 \%$ & $88,17 \%$ & $7,30 \%$ \\
\hline $\begin{array}{c}\text { Peor-30\% } \\
2015\end{array}$ & $0,04 \%$ & $9,05 \%$ & $90,90 \%$ \\
\hline
\end{tabular}

Tabla 6: Matriz de Transición de las variaciones en el ranking entre Jun/15 y Feb/16

La diagonal principal de la tabla 6 nos muestra los porcentajes de hoteles que han permanecido en la misma zona del ranking. El $91,26 \%$ de lo hoteles que en junio de 2015 se encontraban entre los tres primeros deciles (el $30 \%$ mejor), sigue en febrero de 2016 en esa misma zona del ranking. De igual modo el $88,17 \%$ de los que estaban en la zona central y el $90,90 \%$ de los que estaban situados en la parte baja de la clasificación siguen respectivamente en esas mismas zonas al final del periodo analizado.

Es insignificante el porcentaje de hoteles que ha pasado de estar entre los mejores a estar seis meses después entre los peores $(0,04 \%)$. El mismo porcentaje cercano a cero es el de los hoteles que estando entre los peores han conseguido ascender para estar entre los mejores $(0,04 \%)$. De los hoteles en la zona central es algo superior el porcentaje de los que descienden en la clasificación hacia la zona baja $(7,30 \%)$ que los que suben a la zona alta $(4,53 \%)$. 
En cuanto a las ciudades, destacaremos que más del 90\% de los hoteles de París o Londres, estén en la zona superior, central o inferior del ranking siguen en la misma zona seis meses después

Las tres zonas del ranking tienen en promedio, un grado de rigidez muy elevado, siendo del $91,1 \%$ en las zonas altas, del $85,9 \%$ en las zonas medias y del 87,7 en las zonas bajas. La movilidad por tanto entre las distintas zonas de la clasificación es muy baja.

\section{VARIACION EN LAS PUNTUACIONES}

Según indica Tripadvisor en su propia web, (Tripadvisor 2016), su famoso e influyente ranking de hoteles se establece, entre otras variables, en función de las puntuaciones globales que obtienen los hoteles a partir de las valoraciones dadas por los clientes a los distintos establecimientos.

Cada usuario de Tripadvisor, además de realizar comentarios sobre su experiencia en el hotel o subir fotos del establecimiento, puede valorar el hotel con una puntuación que va desde un mínimo de 1 hasta un máximo de 5. A partir de estas puntuaciones individuales, (y mediante un algoritmo secreto) Tripadvisor calcula una valoración global para cada establecimiento que igualmente variará desde el 1 (mínimo) hasta el 5 (máximo). Esta valoración global será determinante para fijar la posición del hotel en el ranking de su ciudad. Es por tanto obligatorio conocer también cómo han evolucionado las puntuaciones de los hoteles entre junio/2015 y febrero/2016, ya que aquí podríamos encontrar una explicación de la falta de movilidad de los rankings.

La tabla 7 recoge la evolución de la puntuación global media. En filas aparecen las puntuaciones de los hoteles en jun/2015 y en columnas las puntuaciones en feb/16

\begin{tabular}{|c|c|c|c|c|c|c|c|c|c|}
\cline { 2 - 10 } \multicolumn{1}{c|}{} & \multicolumn{7}{c|}{ Puntos feb/2016 } \\
\hline $\begin{array}{c}\text { Puntos } \\
\text { jun/2015 }\end{array}$ & 1 & 1,5 & 2 & 2,5 & 3 & 3,5 & 4 & 4,5 & 5 \\
\hline 1 & $78,9 \%$ & $5,3 \%$ & $10,5 \%$ & $0,0 \%$ & $5,3 \%$ & $0,0 \%$ & $0,0 \%$ & $0,0 \%$ & $0,0 \%$ \\
\hline 1,5 & $1,2 \%$ & $88,0 \%$ & $7,2 \%$ & $1,2 \%$ & $2,4 \%$ & $0,0 \%$ & $0,0 \%$ & $0,0 \%$ & $0,0 \%$ \\
\hline 2 & $0,0 \%$ & $1,7 \%$ & $85,5 \%$ & $12,4 \%$ & $0,4 \%$ & $0,0 \%$ & $0,0 \%$ & $0,0 \%$ & $0,0 \%$ \\
\hline 2,5 & $0,0 \%$ & $0,0 \%$ & $1,8 \%$ & $86,5 \%$ & $11,1 \%$ & $0,6 \%$ & $0,0 \%$ & $0,0 \%$ & $0,0 \%$ \\
\hline 3 & $0,0 \%$ & $0,0 \%$ & $0,0 \%$ & $3,8 \%$ & $86,1 \%$ & $10,0 \%$ & $0,0 \%$ & $0,1 \%$ & $0,0 \%$ \\
\hline 3,5 & $0,0 \%$ & $0,0 \%$ & $0,0 \%$ & $0,1 \%$ & $3,0 \%$ & $88,4 \%$ & $8,5 \%$ & $0,1 \%$ & $0,1 \%$ \\
\hline 4 & $0,0 \%$ & $0,0 \%$ & $0,0 \%$ & $0,0 \%$ & $0,1 \%$ & $3,4 \%$ & $93,2 \%$ & $3,3 \%$ & $0,0 \%$ \\
\hline 4,5 & $0,0 \%$ & $0,0 \%$ & $0,0 \%$ & $0,0 \%$ & $0,0 \%$ & $0,0 \%$ & $5,0 \%$ & $94,1 \%$ & $0,9 \%$ \\
\hline 5 & $0,0 \%$ & $0,0 \%$ & $0,0 \%$ & $0,0 \%$ & $0,0 \%$ & $0,0 \%$ & $0,6 \%$ & $15,2 \%$ & $84,2 \%$ \\
\hline
\end{tabular}

Tabla 7: Matriz de Transición de las variaciones en las valoraciones de los hoteles

Como podemos observar, es muy elevado el número de hoteles que mantiene la misma valoración entre el momento inicial y final (Diagonal principal). A excepción de los hoteles con una puntuación media igual a uno, todos los demás porcentajes están por encima del 80\%. Así de los hoteles con un 4 ó un 4,5 el $93.2 \%$ y el $94.1 \%$ respectivamente conservan la misma valoración seis meses después.

Si analizamos ahora en qué medida han aumentado o disminuido las puntuaciones, la tabla siguiente recoge el número total de hoteles para las distintas variaciones en la puntuación: 


\begin{tabular}{|c|c|c|c|}
\hline Dif. Puntos & Hoteles & $\%$ & $\%$ Acum. \\
\hline-1 & 4 & $0,05 \%$ & \multirow{2}{*}{$3,82 \%$} \\
\hline$-0,5$ & 307 & $3,77 \%$ & \\
\hline 0 & 7380 & $90,61 \%$ & $90,61 \%$ \\
\hline 0,5 & 441 & $5,41 \%$ & \multirow{4}{*}{$5,57 \%$} \\
\hline 1 & 8 & $0,10 \%$ & \\
\hline 1,5 & 4 & $0,05 \%$ & \\
\hline 2 & 1 & $0,01 \%$ & \\
\hline Total & 8145 & $100 \%$ & $100 \%$ \\
\hline
\end{tabular}

Tabla 8: Variaciones en la puntuación de los hoteles

Observamos que la gran mayoría de hoteles $(90,61 \%)$ no ha variado su puntuación en el periodo analizado. En los extremos encontramos que tan sólo un hotel ha aumentado su valoración dos puntos, mientras que son cuatro los hoteles que han visto reducida su valoración en 1 punto. Sólo el 5,6\% de los hoteles observados han aumentan su puntuación mientras que es aún menor (el 3,8\%) el número de hoteles que han visto disminuir su valoración.

\section{VARIACION EN EL NUMERO DE OPINIONES}

Las valoraciones individuales de los turistas son, en principio, el elemento determinante para fijar la puntuación global media del hotel, y a partir de esta, establecer la clasificación de los hoteles en cada ciudad. Es por tanto obligatorio analizar cómo ha evolucionado el número de comentarios recibidos por los hoteles.

Como vemos en la tabla 9, el ritmo al que crecen los comentarios en Tripadvisor es muy elevado. En seis meses se han incorporado 702.021 nuevas opiniones, lo que supone un incremento del 23,7\%, pasando de 2.967.160 comentarios en 2015 a 3.669 .181 seis meses después. Si esta variación se ha producido en tan sólo seis meses, podemos concluir que, en este periodo, se han añadido en promedio cada mes 117.000 comentarios en el conjunto de hoteles de la muestra, aproximadamente 3.900 comentarios por día.

\begin{tabular}{|c|c|c|c|c|}
\hline & 2015 & 2016 & Dif & Dif\% \\
\hline Hoteles & 8.145 & 8.145 & 0 & $0,00 \%$ \\
\hline Total Opiniones & 2.967 .160 & 3.669 .181 & 702.021 & $23,66 \%$ \\
\hline Promedio opiniones por hotel & 364,29 & 450,48 & 86 & $23,66 \%$ \\
\hline Máximo opiniones por hotel & 8.399 & 10.909 & 2.510 & $29,88 \%$ \\
\hline Mínimo opiniones por hotel & 2 & 2 & 0 & $0,00 \%$ \\
\hline
\end{tabular}

Tabla 9: № de comentarios

Por último, queremos mostrar en el siguiente gráfico como el tamaño de la ciudad no parece afectar al número de comentarios que en promedio reciben los hoteles. Así Dublín, y La Valeta son las dos ciudades que por término medio más comentarios tienen por hotel (929 y 853 respectivamente), muy superiores a grandes capitales como Roma y París (con 371 y 353 comentarios por hotel). 


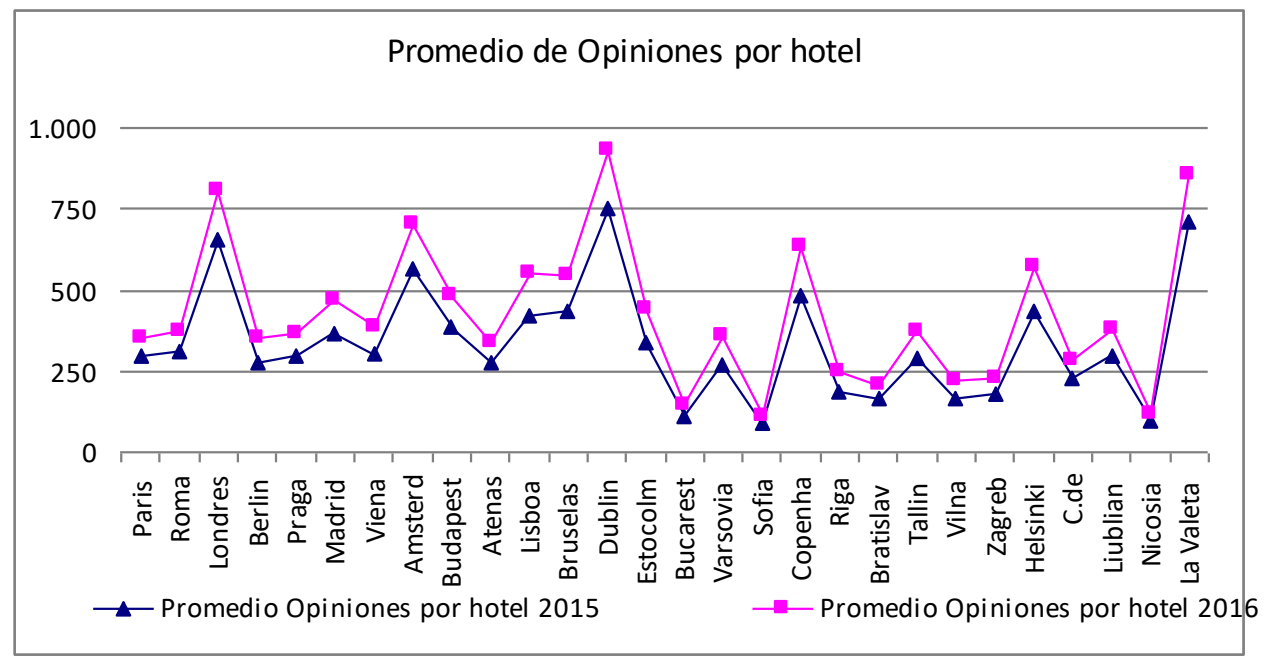

Gráfico 1: Número de comentarios (promedio) por hotel y ciudad

\section{CONCLUSIONES}

Nuestro análisis de más de 8.000 hoteles pertenecientes a las 28 capitales europeas nos ha permitido comprobar la enorme popularidad de Tripadvisor ya que en tan sólo seis meses hemos encontrado más de 700.000 nuevos comentarios añadidos por los usuarios para los hoteles de la muestra, lo que supone un incremento del $24 \%$ en tan breve periodo de tiempo. Es evidente la capacidad de Tripadvisor para generar nuevas valoraciones y comentarios de los turistas. Una página web que consigue que sus usuarios, sin recibir nada a cambio escriban tal cantidad de comentarios no puede calificarse más que de extraordinaria.

Pero sorprendentemente, esta inmensa cantidad de opiniones de los turistas, apenas tiene reflejo ni en las puntuaciones ni en los rankings de los hoteles de las ciudades analizadas.

Nuestro análisis muestra que, en primer lugar, el elevado número de comentarios apenas modifican la valoración media de los hoteles. La gran mayoría de hoteles $(90,6 \%)$ no ha modificado su puntuación en el periodo analizado. Las 700.000 nuevas opiniones sólo han servido para modificar la valoración del 9\% de hoteles.

En segundo lugar, y dado que el ranking se configura en función de las puntuaciones de los distintos hoteles, si estas no varían, tampoco van a variar las posiciones en la clasificación. Así, aunque se observa una gran Volatilidad, ya que la gran mayoría de los hoteles $(96,59 \%)$ ha variado su posición en el ranking, hay por el contrario muy poca Flexibilidad ya que estas variaciones son muy pequeñas $(4,9 \%$ en las subidas y $4,4 \%$ en las bajadas).

En consecuencia, la Relevancia de estas variaciones, en términos de alcanzar mejores puestos en la clasificación es insignificante. Así, de los hoteles situados en el $30 \%$ superior, en el $40 \%$ central y en el $30 \%$ inferior del ranking, el $91,3 \%$, el $88,2 \%$ y el $90,9 \%$ siguen estando respectivamente en las mismas zonas seis meses después. Hemos comprobado además que estas cifras son muy similares en la gran mayoría de las 28 capitales europeas, sin apenas excepciones.

Por último, el 78\% de los hoteles situados en el Top 5, seguía ahí seis meses después. Y el 84\% de los situados en el Top 10 ó el 85\% de los situados en el Top 30 también seguían en la misma zona seis meses después. Porcentajes similares encontramos también en las zonas bajas. De los hoteles situados entre los cinco peores de todas las capitales europeas, el 73,5 seguía estando entre los peores seis meses después. El 75,9\% de los 10 peores o el 85,4\% de los 30 peores también seguían entre los peores en febrero de 2016.

Por tanto, como primera conclusión debemos señalar que, al no variar las puntuaciones globales de los hoteles, el ranking de Tripadvisor ha llegado a una situación de estado estacionario, en el que apenas se producen cambios relevantes en la clasificación.

¿Por qué esta gran cantidad de comentarios no ha provocado cambios en las puntuaciones de los hoteles y en consecuencia tampoco en las posiciones en el ranking? La respuesta en nuestra opinión, está en las propias características de Tripadvisor.

1- Unas opciones de puntuación muy limitadas. Sólo hay 9 opciones para puntuar. (1 - 1,5 - 2 - 2,5 - 3 - 3,5 - 4 $4,5-5)$. 
2- Una gran cantidad de opiniones que se añaden día a día, con una puntuación muy similar, concentrándose la mayoría de ellas en una zona media (el $76,4 \%$ de los hoteles tienen una puntuación media que se sitúa entre el 3,5 y el 4,5). Hay por tanto muy pocas puntuaciones extremas. Como hemos estudiado en un trabajo previo, (Molinillo et al, 2016) la mayoría de las puntuaciones de los turistas son altas o medias, sin apenas puntuaciones negativas que puedan hacer cambiar de forma significativa la valoración global media de un hotel.

Si la mayoría de los hoteles tienen unas puntuaciones medias muy similares (entre 3,5 y el 4,5), y la mayoría de las puntuaciones de los nuevos comentarios están también en estos niveles, con muy pocos casos de puntuaciones bajas, es por tanto prácticamente imposible dado el elevado número de comentarios, que la puntuación final media de los hoteles se modifique a lo largo del tiempo, por muchos comentarios nuevos que se añadan.

Son estas circunstancias, algunas de ellas fruto del éxito alcanzado por Tripadvisor, así como por la profesionalidad y buen hacer de los hoteleros, las que han provocado que el ranking que debe ser en definitiva una herramienta útil para tomar decisiones, apenas varíe y por consiguiente esté perdiendo gran parte de su atractivo y utilidad.

Parece razonable pensar que al venir esta falta de movilidad provocada en gran medida por las propias características de Tripadvisor, pudiera existir una intención deliberada por parte de esta empresa para que el ranking apenas cambie.

Dejando planteada esta posibilidad para futuros debates con investigadores y profesionales del sector, creemos en cualquier caso que sería un grave error tratar de reducir o limitar la movilidad en la clasificación, pues sin entrar en consideraciones éticas, se estaría en primer lugar desincentivando a los usuarios para que aporten sus comentarios y valoraciones (si nada va a cambiar, ¿para qué escribir comentarios, para qué puntuar un hotel? Además, se estaría también trasladando a los hoteleros la idea de que por más que hagan para mejorar la posición de su hotel en la clasificación, sus posibilidades de estar algún día entre los mejores son mínimas, casi nulas.

Por ello, y considerando que cierto nivel de movilidad es recomendable, proponemos dos modificaciones en el sistema de Tripadvisor para recuperar el dinamismo que todo buen ranking debe tener y que no suponen más que una mínima alteración del diseño actual del servicio:

1. En primer lugar recomendamos ampliar el rango de puntuaciones posibles, manteniendo la puntuación mínima en 1 , pero aumentando la puntuación máxima desde el 5 actual al 10.

2. En segundo lugar, proponemos aumentar las opciones de puntuación disponibles. Si actualmente entre, por ejemplo, un 3 y un 4 , sólo se admite puntuar con un 3,5, proponemos que se amplíen las opciones para puntuar por décimas de punto $(\ldots 3-3,1-3,2-3,3-\ldots 3,8-3,9-4 \ldots)$

De este modo, con estas dos modificaciones, se ampliarían las opciones para puntuar un hotel desde las 9 actuales a más de 90, lo que aumentaría la dispersión de las puntuaciones y evitaría encontrarnos con tantos hoteles con la misma valoración global. Sería entonces más fácil que se produjeran variaciones en las puntuaciones medias de los hoteles y por pequeñas que éstas fueran provocarían un mayor número de cambios en las posiciones dentro del ranking.

Como todo trabajo de investigación, este adolece de ciertas debilidades y limitaciones. En primer lugar, y aunque para un periodo de tiempo relativamente corto (seis meses) los datos son suficientemente contundentes para extraer una primera aproximación al problema de la falta de movilidad de los rankjngs, creemos que sería conveniente ampliar el horizonte temporal al menos a un año. Esto enriquecería y fortalecería las conclusiones expuestas. En segundo lugar, si bien el estudio tiene un marcado carácter internacional, debemos reconocer la limitación que supone haber analizado exclusivamente hoteles de capitales europeas, por lo que será necesario analizar adicionalmente hoteles de otras partes del mundo, y también de otra tipología de clientes (hoteles en destinos de sol y playa, en ciudades de provincia...). Como líneas futuras de investigación sería muy interesante ampliar el estudio a otros tipos de establecimientos (restaurantes) así como a otras webs (booking.com).

Por último, consideramos como la aportación principal de este trabajo el abrir un nuevo campo de investigación y debate hasta ahora inexplorado (la Movilidad de los Rankings), con aplicaciones en cualquier actividad donde un ranking tenga poder o influencia.

\section{BIBLIOGRAFIA}

Biffaro, L. (2015). Unfair Commercial Practices and Online Consumer Reviews: the Italian Tripadvisor Case. Rivista Italiana di Antitrust/Italian Antitrust Review,2(1). 
Callarisa-Fiol, L. J., Sánchez-García, J., Moliner Tena, M. Á., \& Forgas-Coll, S. (2012). La importancia de las comunidades virtuales para el analisis del valor de marca.el caso de tripadvisor en hong kong y paris. Papers de turisme, (54), 89-115.

Chen, Y. F., \& Law, R. (2016). A Review of Research on Electronic Word-of-Mouth in Hospitality and Tourism Management. International Journal of Hospitality \& Tourism Administration, 17(4), 347-372.

Eco, U., \& Irazazábal, M. P. (2009). El vértigo de las listas. Lumen Editorial.

European Commission (2014). Study on Online Consumer Reviews in the Hotel Sector. Final report.Disponible en : http://bookshop.europa.eu/en/study-on-online-consumer-reviews-in-the-hotel-sector-pbND0414464/

Ghose, a., Ipeirotis, P. G., \& Li, B. (2012). Designing Ranking Systems for Hotels on Travel Search Engines by Mining User-Generated and Crowdsourced Content. Marketing Science, 31(3), 493-520. doi:10.1287/mksc. 1110.0700

Hensens,W. (2010). The reliability of data from guest reviews on Tripadvisor as a contemporary social media platform. Paper submitted and presented at SAIMS 2010 in Mpekweni Resort.

Jain, D., Juman, D., Quinby, D., \& Rauch, M. (2012). Social Media in Travel 2012. Social Networks \& Traveler Reviews. PhocusWright.

Jurca, R., Garcin, F., Talwar, A., \& Faltings, B. (2010). Reporting incentives and biases in online review forums. ACM Transactions on the Web, 4(2), 1-27. doi:10.1145/1734200.1734202

Mateos, J. C., Martín, M. Á. R., \& Martín-Velicia, F. A. (2014). Disrupción en el uso de la web 2.0 en los hoteles independientes. In Cultura, desarrollo y nuevas tecnologías: VII Jornadas de Investigación en Turismo, Sevilla, 11 y 12 de Junio de 2014 (pp. 169-183).

Melián González, S., Bulchand Gidumal, J., \& González López-Valcárcel, B. (2010). la participación de los clientes en sitios web de valoración de servicios turístico.El caso de TripAdvisor. Análisis Turístico, (2o semestre), 17-22.

Mellinas, J. P., María-Dolores, S. M. M., \& García, J. J. B. (2015). Booking. com: The unexpected scoring system. Tourism Management, 49, 72-74.

Mistilis, N., \& Buhalis, D. (2012). Challenges and potential of the Semantic Web for tourism. E-review of Tourism Research, 10(2), 51-55. Retrieved from http://search.ebscohost.com/login.aspx?direct=true\&db=hjh\&AN=84339717\&lang=es\&site=ehostlive\&scope $=$ site

Molinillo, S. Ximénez-de-Sandoval, J.L., Fernández-Morales, A. \& Stefaniak,A.C. (2016). Hotel assessment through social media-Tripadvisor as a case stydy.Encontros Científicos-Tourism Management Studies, 12(1), 15-24

O'Mahony, M. P., \& Smyth, B. (2009). Learning to recommend helpful hotel reviews. Proceedings of the third ACM conference on Recommender systems - RecSys '09, 305. doi:10.1145/1639714.1639774

Ong, B. S. (2012). The Perceived Influence of User Reviews in the Hospitality Industry. Journal of Hospitality Marketing \& Management, 21(5), 463-485. doi:10.1080/19368623.2012.626743

Ott, M., Cardie, C., \& Hancock, J. (2012). Estimating the Prevalence of Deception in Online Review Communities. Proceedings of the 21st international conference on World Wide Web WWW 12, 201-210. Retrieved from http://dl.acm.org/citation.cfm?doid=2187836.2187864

Ott, M., Choi, Y., Cardie, C., \& Hancock, J. T. (2011). Finding Deceptive Opinion Spam by Any Stretch of the Imagination. Computational Linguistics, 17(June 19-24, 2011), 11. Retrieved from http://arxiv.org/abs/1107.4557

Scott, S. V, \& Orlikowski, W. J. (2012). Reconfiguring relations of accountability: Materialization of social media in the travel sector. Accounting, Organizations and Society, 37(1), 26-40. doi: 10.1016/j.aos.2011.11.005

Smyth, B., Wu, G., \& Greene, D. (2010). Does TripAdvisor Makes Hotels Better?. Technical Report, (08).

Stringam, B. B., Gerdes, J., \& Vanleeuwen, D. M. (2010). Assessing the Importance and Relationships of Ratings on User-Generated Traveler Reviews. Journal of Quality Assurance in Hospitality \& Tourism, 11(2), 7392. doi:10.1080/1528008X.2010.482000

Sotiriadis, M. D., \& Sotiriadis, M. D. (2017). Sharing tourism experiences in social media: A literature review and a set of suggested business strategies.International Journal of Contemporary Hospitality Management, 29(1), 179-225.

TripAdvisor. (2016). Changes to the Tripadvisor Popularity Ranking Algorithm. Disponible en: https://www.tripadvisor.com/TripAdvisorlnsights/n2701/changes-tripadvisor-popularity-ranking-algorithm 
Verma, R., Stock, D., \& McCarthy, L. (2012). Customer Preferences for Online, Social Media, and Mobile Innovations in the Hospitality Industry. Cornell Hospitality Quarterly, 53(3), 183-186. doi:10.1177/1938965512445161

Wu, G., Greene, D., \& Cunningham, P. (2010). Merging multiple criteria to identify suspicious reviews. Proceedings of the fourth ACM conference on Recommender systems - RecSys '10, 241. doi:10.1145/1864708.1864757

Xiang, Z., \& Gretzel, U. (2010). Role of social media in online travel information search. Tourism Management, 31(2), 179-188. Retrieved from 10.1016/j.tourman.2009.02.016 